\title{
Dynamics of Generalized Tachyon Field in Teleparallel Gravity
}

\author{
Behnaz Fazlpour ${ }^{1}$ and Ali Banijamali ${ }^{2}$ \\ ${ }^{1}$ Department of Physics, Islamic Azad University, Babol Branch, Babol, Iran \\ ${ }^{2}$ Department of Basic Sciences, Babol University of Technology, Babol 47148-71167, Iran \\ Correspondence should be addressed to Behnaz Fazlpour; b.fazlpour@umz.ac.ir
}

Received 11 October 2014; Accepted 15 January 2015

Academic Editor: Chao-Qiang Geng

Copyright (C) 2015 B. Fazlpour and A. Banijamali. This is an open access article distributed under the Creative Commons Attribution License, which permits unrestricted use, distribution, and reproduction in any medium, provided the original work is properly cited. The publication of this article was funded by SCOAP ${ }^{3}$.

\begin{abstract}
We study dynamics of generalized tachyon scalar field in the framework of teleparallel gravity. This model is an extension of tachyonic teleparallel dark energy model which has been proposed by Banijamali and Fazlpour (2012). In contrast with tachyonic teleparallel dark energy model that has no scaling attractors, here we find some scaling attractors which means that the cosmological coincidence problem can be alleviated. Scaling attractors are presented for both interacting and noninteracting dark energy and dark matter cases.
\end{abstract}

\section{Introduction}

The usual proposal to explain the late-time accelerated expansion of our universe is an unknown energy component, dubbed as dark energy. The natural choice and most attractive candidate for dark energy is the cosmological constant, but it is not well accepted because of the cosmological constant problem $[1,2]$ as well as the age problem [3]. Thus, many dynamical dark energy models as alternative possibilities have been proposed. Quintessence, phantom, k-essence, quintom, and tachyon field are the most familiar dark energy models in the literature (for reviews on dark energy models see $[4,5])$. The tachyon field arising in the context of string theory $[4,5]$ and its application in cosmology both as a source of early inflation and late-time cosmic acceleration have been extensively studied [6-9].

The so-called "teleparallel equivalent of general relativity" or teleparallel gravity was first constructed by Einstein [1013]. In this formulation one uses the curvature-less Weitzenbock connection instead of the torsion-less Levi-Civita connection. The relevant Lagrangian in teleparallel gravity is the torsion scalar $T$ which is constructed by contraction of the torsion tensor. We recall that the Einstein-Hilbert Lagrangian $R$ is constructed by contraction of the curvature tensor. Since teleparallel gravity with torsion scalar as Lagrangian density is completely equivalent to a matter-dominated universe in the framework of general relativity, it cannot be accelerated. Thus one should generalize teleparallel gravity either by replacing $T$ with an arbitrary function, the so-called $f(T)$ gravity [14-16], or by adding dark energy into teleparallel gravity allowing also a nonminimal coupling between dark energy and gravity. Note that both approaches are inspired by the similar modifications of general relativity, that is, $f(R)$ gravity $[17,18]$ and nonminimally coupled dark energy models in the framework of general relativity [19-26].

Recently Geng et al. [27, 28] have included a nonminimal coupling between quintessence and gravity in the context of teleparallel gravity. This theory has been called "teleparallel dark energy" and its dynamics was studied in [29-31]. Tachyonic teleparallel dark energy is a generalization of teleparallel dark energy by inserting a noncanonical scalar field instead of quintessence in the action [32]. Phase-space analysis of this model has been investigated in [33]. On the other hand, there is no physical argument to exclude the interaction between dark energy and dark matter. The interaction between these completely different components of our universe has the same important consequences such as addressing the coincidence problem [34]. There are also observational evidences of the interaction in dark sector [35-40]. In [35-38] Bertolami et al. have shown that this interaction might imply a violation of the equivalence principle. By using optical, X-ray, and weak lensing data from the relaxed galaxy clusters, Abdalla et al. 
$[39,40]$ have found the signature of interaction between dark energy and dark matter. An interacting scenario provides solution to a number of cosmological problems in both canonical scalar field models [41] and nonminimally coupled scalar field models such as Brans-Dick field [42, 43].

In this paper, we consider generalized tachyon field as responsible for dark energy in the framework of teleparallel gravity. We will be interested in performing a dynamical analysis of such a model in FRW space time. In such a study we investigate our model for both interacting and noninteracting cases. We consider the nonminimal coupling function of the form $f(\phi) \propto \phi^{2}$ and choose $n=2$ in (2). The basic equations are presented in Section 2. In Section 3 the evolution equations are translated in the language of the autonomous dynamical system by suitable transformation of the basic variables. Section 3.1 deals with phase-space analysis as well as the cosmological implications of the equilibrium points of the model in noninteracting dark energy dark matter case. In Section 3.2 an interaction between dark energy and dark matter has been considered and critical points and their behavior have been extracted. Section 4 is devoted to a short summary of our results.

\section{Basic Equations}

Our model is described by the following action as a generalization of tachyon teleparallel dark energy model [32]:

$$
\begin{gathered}
S=\int d^{4} x e\left[\mathscr{L}_{T}+\mathscr{L}_{\varphi}+\mathscr{L}_{m}\right], \\
\mathscr{L}_{T}=\frac{T}{2 \kappa^{2}}, \\
\mathscr{L}_{\varphi}=\xi f(\varphi) T-V(\varphi)(1-2 X)^{n},
\end{gathered}
$$

where $e=\operatorname{det}\left(e_{\mu}^{i}\right)=\sqrt{-g}\left(e_{\mu}^{i}\right.$ are the orthonormal components of the tetrad), while $T / 2 \kappa^{2}$ is the Lagrangian of teleparallelism with $T$ as the torsion scalar (for an introductory review of teleparallelism see [12]). $\mathscr{L}_{\varphi}$ shows nonminimal coupling of generalized tachyon field $\varphi$ with gravity in the framework of teleparallel gravity and $X=(1 / 2) \partial_{\mu} \varphi \partial^{\mu} \varphi$. The second part in $\mathscr{L}_{\varphi}$ is the Lagrangian density of the generalized tachyon field which has been studied in [44, 45]. $f(\varphi)$ is the nonminimal coupling function, $\xi$ is a dimensionless constant measuring the nonminimal coupling, and $\mathscr{L}_{m}$ is the matter Lagrangian. For $n=1 / 2$ our model reduced to tachyonic teleparallel dark energy discussed in [32]. Here we consider the case $n=2$ for two reasons. The first is that for arbitrary $n$ our equations will be very complicated and one can not solve them analytically and the second is that for $n=2$ we will obtain interesting physical results as we will see below.

Furthermore, due to complexity of tachyon dynamics, [46] has proposed an approach based on the redefinition of the tachyon field as follows:

$$
\varphi \longrightarrow \phi=\int d \varphi \sqrt{V(\varphi)} \Longleftrightarrow \partial \varphi=\frac{\partial \phi}{\sqrt{V(\phi)}} .
$$

In order to obtain a closed autonomous system and perform the phase-space analysis of the model, we apply (3) in (2) for $n=2$ that leads to the following action:

$$
S=\int d^{4} x e\left[\frac{T}{2 \kappa^{2}}+\xi f(\phi) T-V(\phi)\left(1-\frac{2 X}{V(\phi)}\right)^{2}+\mathscr{L}_{m}\right]
$$

In a spatially flat FRW space-time,

$$
d s^{2}=d t^{2}-a^{2}(t)\left(d r^{2}+r^{2} d \Omega^{2}\right)
$$

and a vierbein choice of the form $e_{\mu}^{i}=\operatorname{diag}(1, a, a, a)$, the corresponding Friedmann equations are given by

$$
\begin{gathered}
H^{2}=\frac{1}{3}\left(\rho_{\phi}+\rho_{m}\right), \\
\dot{H}=-\frac{1}{2}\left(\rho_{\phi}+P_{\phi}+\rho_{m}+P_{m}\right),
\end{gathered}
$$

where $H=\dot{a} / a$ is the Hubble parameter and a dot stands for the derivative with respect to the cosmic time $t$. In these equations, $\rho_{m}$ and $P_{m}$ are the matter energy density and pressure, respectively.

The effective energy density and pressure of generalized tachyon dark energy read

$$
\begin{aligned}
\rho_{\phi} & =V(\phi)+2 \dot{\phi}^{2}-3 \frac{\dot{\phi}^{4}}{V(\phi)}-6 \xi H^{2} f(\phi), \\
P_{\phi}= & -V(\phi)+2 \xi\left(3 H^{2}+2 \dot{H}\right) f(\phi)+10 \xi H f_{, \phi} \dot{\phi} \\
& +\dot{\phi}^{2}\left(2-\frac{\dot{\phi}^{2}}{V(\phi)}\right),
\end{aligned}
$$

where $f_{, \phi}=d f / d \phi$.

The equation of motion of the scalar field can be obtained by variation of the action (4) with respect to $\phi$ :

$$
\begin{aligned}
\ddot{\phi} & +3 \mu^{-2} \nu^{2} H \dot{\phi}+\frac{1}{4} \nu^{2}\left(1+\frac{3 \dot{\phi}^{4}}{V^{2}(\phi)}\right) V_{, \phi} \\
& +6 \xi \nu^{2} H^{2} f_{, \phi}=-\frac{\nu^{2} Q}{4 \dot{\phi}},
\end{aligned}
$$

with $Q$ a general interaction coupling term between dark energy and dark matter, $\mu=1 / \sqrt{1-2 X / V}$, and $\nu=$ $1 / \sqrt{1-6 X / V}$. In (7) and (8) we have used the useful relation:

$$
T=-6 H^{2},
$$

which simply arises from the calculation of torsion scalar for the FRW metric (5). The scalar field evolution (8) expresses the continuity equation for the field and matter as follows:

$$
\begin{aligned}
& \dot{\rho}_{\phi}+3 H\left(1+\omega_{\phi}\right) \rho_{\phi}=-Q, \\
& \dot{\rho}_{m}+3 H\left(1+\omega_{m}\right) \rho_{m}=Q,
\end{aligned}
$$


TABLE 1: Location of the critical points and the corresponding values of the dark energy density parameter $\Omega_{\phi}$ and equation of state $\omega_{\phi}$ and the condition required for an accelerating universe for $Q=0$. Here $\lambda_{1}=\alpha\left(4 \alpha \xi+\sqrt{16 \alpha^{2} \xi^{2}-2 \lambda^{2} \xi}\right) / \lambda^{2}$ and $\lambda_{2}=\alpha\left(4 \alpha \xi-\sqrt{16 \alpha^{2} \xi^{2}-2 \lambda^{2} \xi}\right) / \lambda^{2}$.

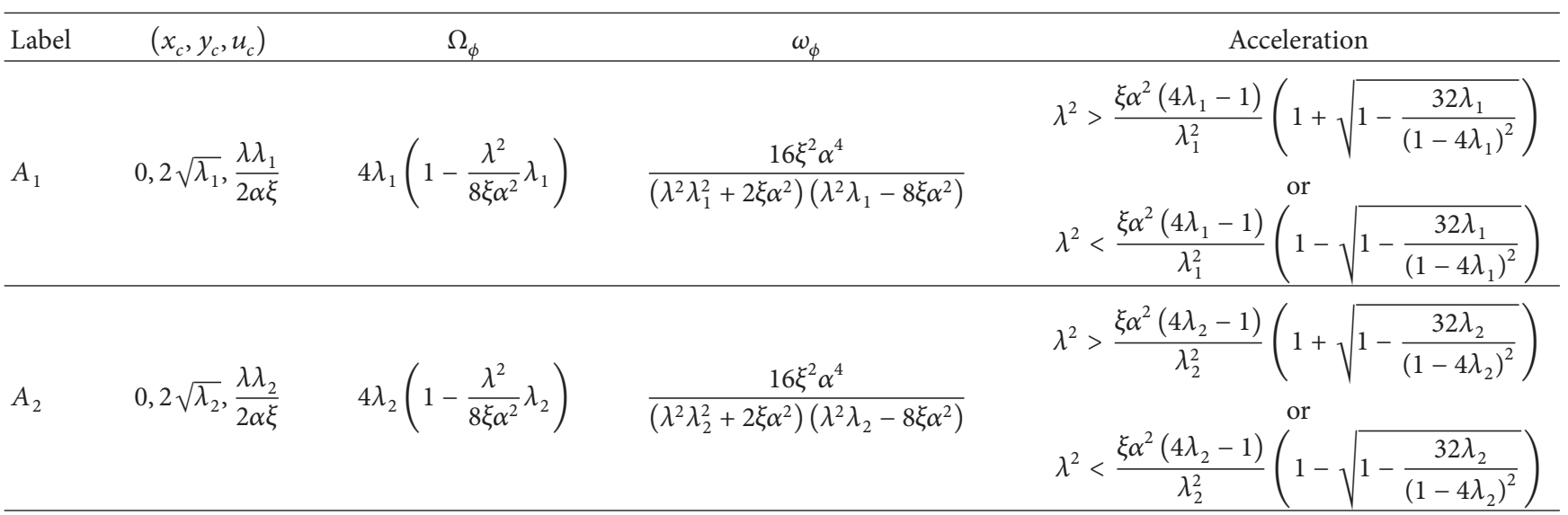

TABLE 2: Stability and existence conditions of the critical points of the model for $Q=0$.

\begin{tabular}{ccc}
\hline Label & Stability & Existence \\
\hline \multirow{4}{*}{$A_{1}$} & Saddle point & For all $\xi<0$ \\
& if $\alpha>0$ and $\xi / \lambda>0$ and & or \\
& stable point & $\xi \geq \lambda^{2} / 8 \alpha^{2}$ \\
\hline & if $\xi>0, \alpha<0$ and $\lambda<0$ & For all $\xi<0$ \\
$A_{2}$ & Saddle point & or \\
& if $\alpha<0$ and $\xi / \lambda<0$ and & stable point \\
& if $\xi>0, \alpha>0$ and $\lambda>0$ & $\xi \geq \lambda^{2} / 8 \alpha^{2}$ \\
\hline
\end{tabular}

where $\omega_{\phi}=P_{\phi} / \rho_{\phi}$ is the equation of state parameter of dark energy which is attributed to the scalar field $\phi$. The barotropic index is defined by $\gamma \equiv 1+\omega_{m}$ with $0<\gamma<2$.

Although dynamics of tachyonic teleparallel dark energy has been studied in [33], no scaling attractors has been found. Here we are going to perform a phase-space analysis of generalized tachyonic teleparallel dark energy and as we will see below that some interesting scaling attractors appear in such theory.

\section{Cosmological Dynamics}

In order to perform phase-space and stability analysis of the model, we introduce the following auxiliary variables:

$$
x \equiv \frac{\dot{\phi}}{\sqrt{V}}, \quad y \equiv \frac{\sqrt{V}}{\sqrt{3} H}, \quad u \equiv \sqrt{f} .
$$

The auxiliary variables allow us to straightforwardly obtain the density parameter of dark energy and dark matter:

$$
\begin{gathered}
\Omega_{\phi} \equiv \frac{\rho_{\phi}}{3 H^{2}}=\mu^{-2} y^{2}\left(1+3 x^{2}\right)-2 \xi u^{2}, \\
\Omega_{m} \equiv \frac{\rho_{m}}{3 H^{2}}=1-\Omega_{\phi},
\end{gathered}
$$

while the equation of state of the field reads

$$
\begin{aligned}
\omega_{\phi} & \equiv \frac{P_{\phi}}{\rho_{\phi}} \\
& =\frac{-\mu^{-4} y^{2}+2 \xi u[(5 \sqrt{3} / 3) \alpha x y+u(1-(2 / 3) s)]}{\mu^{-2} y^{2}\left(1+3 x^{2}\right)-2 \xi u^{2}},
\end{aligned}
$$

where $\alpha \equiv f_{, \phi} / \sqrt{f}$ and

$$
\begin{aligned}
s=-\frac{\dot{H}}{H^{2}}= & \left(2 \xi u^{2}+1\right)^{-1} \\
\cdot & {\left[5 \sqrt{3} \alpha \xi u x y+6 \mu^{-2} x^{2} y^{2}\right.} \\
& \left.-\frac{3}{2} \gamma \mu^{-2} y^{2}\left(1+3 x^{2}\right)\right]+\frac{3 \gamma}{2} .
\end{aligned}
$$

From now we concentrate on exponential scalar field potential of the form $V=V_{0} e^{-k \lambda \phi}$ and the nonminimal coupling function of the form $f(\phi) \propto \phi^{2}$. These choices lead to constant $\lambda$ and $\alpha$, respectively.

Another quantities with great physical significance, namely, the total equation of state parameter and the deceleration parameter, are given by

$$
\begin{aligned}
& \omega_{\text {tot }} \equiv \frac{P_{\phi}+P_{m}}{\rho_{\phi}+\rho_{m}}=\mu^{-2} y^{2}\left(4 x^{2}-\gamma\left(1+3 x^{2}\right)\right) \\
&+2 \xi u\left[\frac{5 \sqrt{3}}{3} \alpha x y+u\left(\gamma-\frac{2}{3} s\right)\right] \\
&+\gamma-1, \\
& q \equiv-1-\frac{\dot{H}}{H^{2}}= \frac{1}{2}+\frac{3}{2} \omega_{\text {tot }} \\
&=\frac{3}{2} \mu^{-2} y^{2}\left(4 x^{2}-\gamma\left(1+3 x^{2}\right)\right) \\
&+\xi u[5 \sqrt{3} \alpha x y+u(3 \gamma-2 s)]+\frac{3 \gamma}{2}-1 .
\end{aligned}
$$




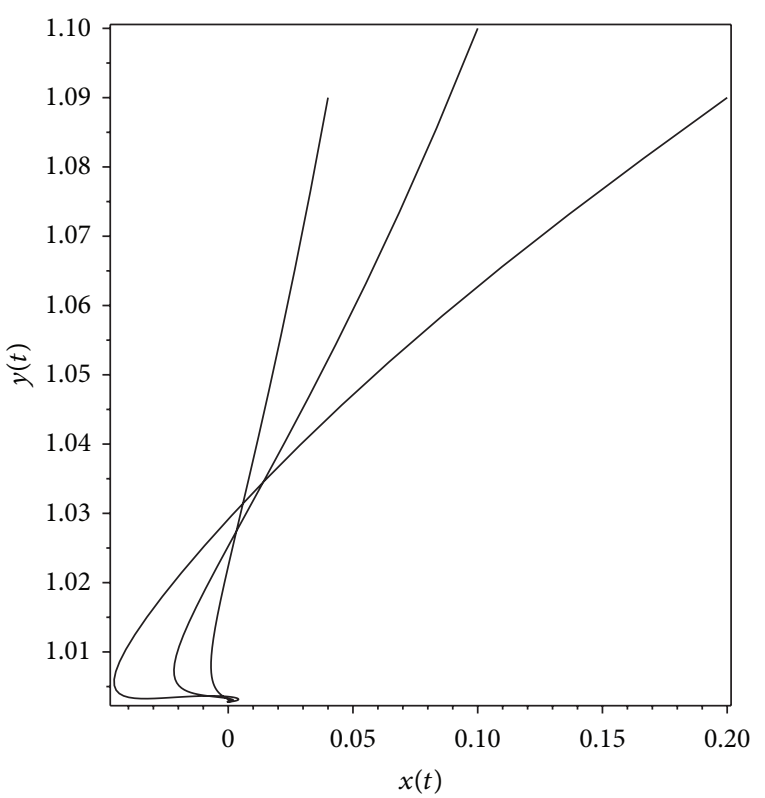

(a)

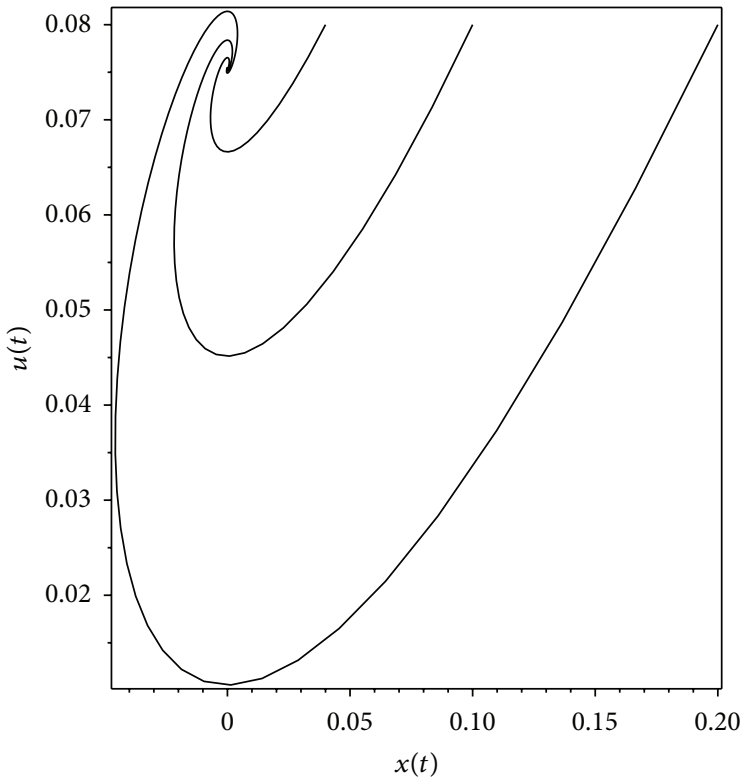

(b)

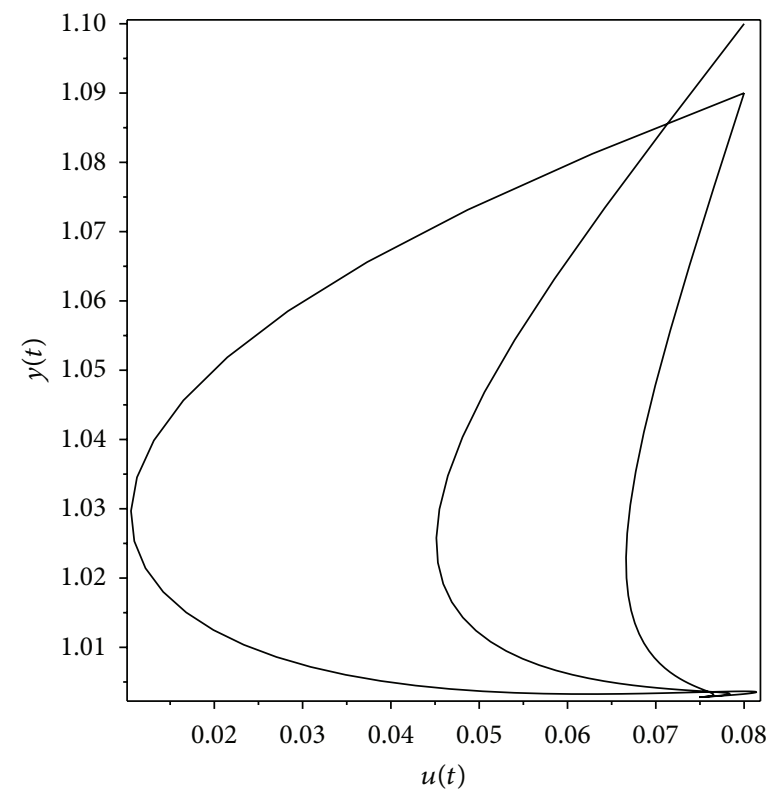

(c)

Figure 1: From (a) to (c), the projections of the phase-space trajectories on the $x-y, x-u$, and $u-y$ planes with $\xi=0.5, \lambda=-0.6$, and $\alpha=-2$ for $Q=0$. For these values of the parameters, point $A_{1}$ is a stable attractor of the model.

Using auxiliary variables (11) the evolution equations (6) and (8) can be recast as a dynamical system of ordinary differential equations:

$$
\begin{aligned}
x^{\prime}=\frac{\sqrt{3}}{2}[ & \lambda x^{2} y+\frac{1}{2} \lambda \nu^{2}\left(1+3 x^{4}\right) y \\
& \left.-4 \alpha \xi \nu^{2} u y^{-1}-2 \sqrt{3} \mu^{-2} v^{2} x\right]-\widehat{Q},
\end{aligned}
$$

$$
\begin{aligned}
& y^{\prime}=\left(-\frac{\sqrt{3}}{2} \lambda x y+s\right) y, \\
& u^{\prime}=\frac{\sqrt{3} \alpha x y}{2},
\end{aligned}
$$

where $\widehat{Q}=Q / \dot{\phi} H \sqrt{V(\phi)}, \lambda \equiv-V_{, \phi} / k V$, and prime in (18) denotes differentiation with respect to the so-called e-folding time $N=\ln a$. 


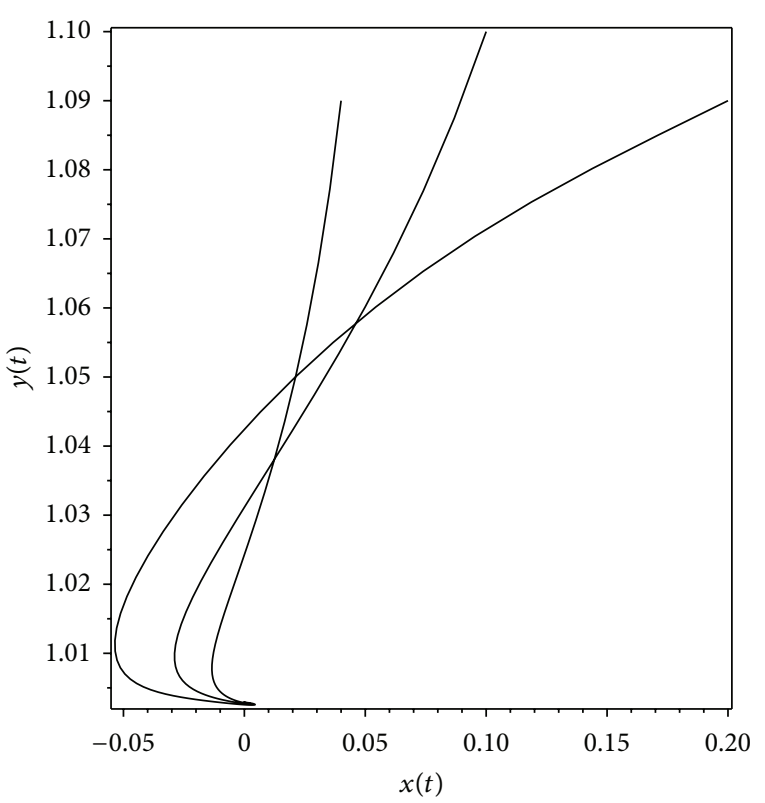

(a)

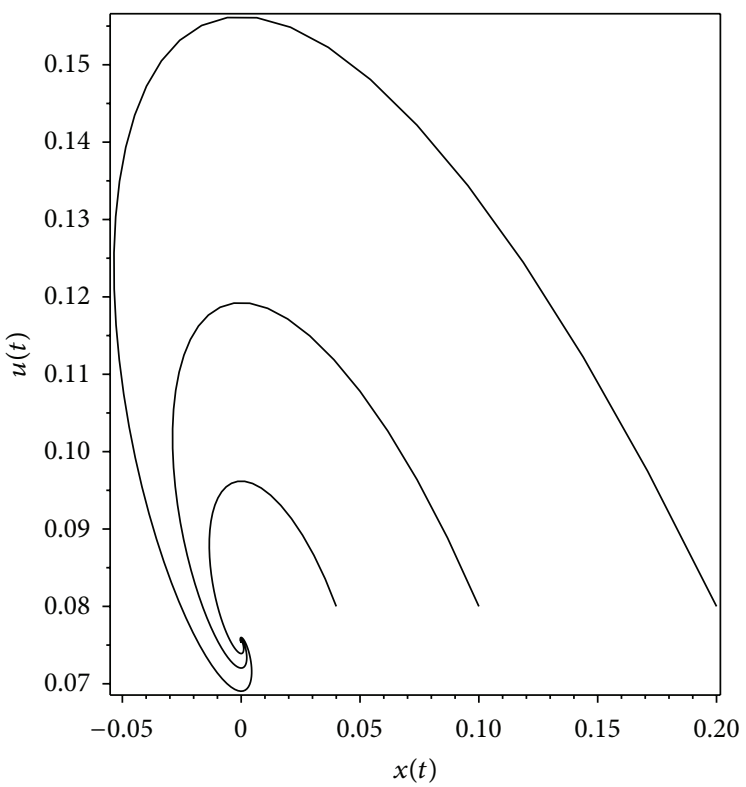

(b)

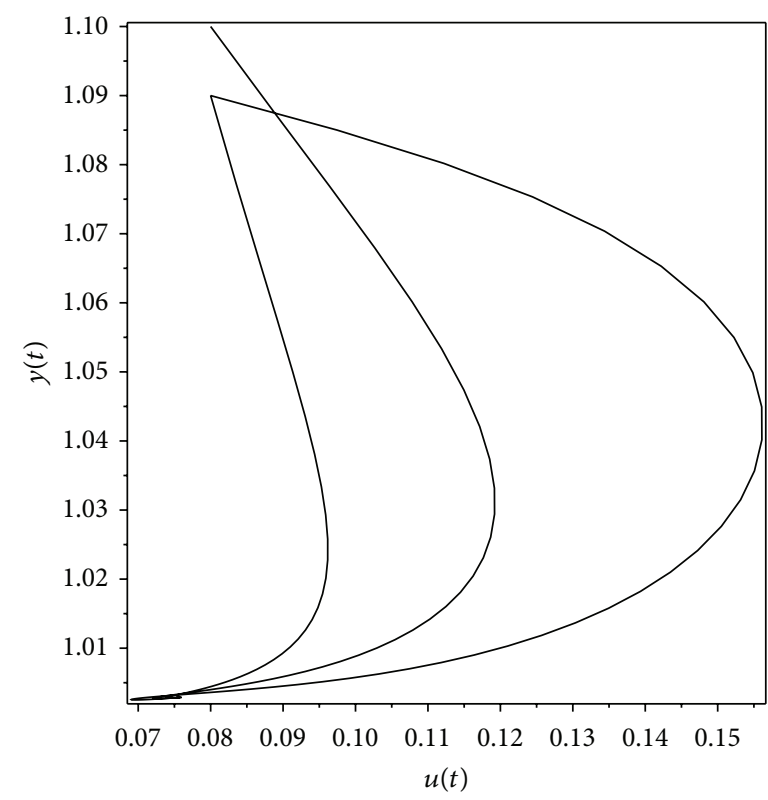

(c)

Figure 2: From (a) to (c), the projections of the phase-space trajectories on the $x-y, x-u$, and $u-y$ planes with $\xi=0.5, \lambda=0.6$, and $\alpha=2$ for $Q=0$. For these values of the parameters, point $A_{2}$ is a stable attractor of the model.

The next step is the introduction of interaction term $Q$ to obtain an autonomous system out of (18). The fixed points $\left(x_{c}, y_{c}, u_{c}\right)$ for which $x^{\prime}=y^{\prime}=u^{\prime}=0$ depend on the choice of the interaction term $Q$ and two general possibilities will be treated in the sequel. The stability of the system at a fixed point can be obtained from the analysis of the determinant and trace of the perturbation matrix $M$. Such a matrix can be constructed by substituting linear perturbations $x \rightarrow$ $x_{c}+\delta x, y \rightarrow y_{c}+\delta y$, and $u \rightarrow u_{c}+\delta u$ about the critical point $\left(x_{c}, y_{c}, u_{c}\right)$ into the autonomous system (18). The
$3 \times 3$ matrix $M$ of the linearized perturbation equations of the autonomous system is shown in the appendix. Therefore, for each critical point we examine the sign of the real part of the eigenvalues of $M$. According to the usual dynamical system analysis, if the eigenvalues are real and have opposite signs, the corresponding critical point is a saddle point. A fixed point is unstable if the eigenvalues are positive and it is stable for negative real part of the eigenvalues.

In the following subsections we will study the dynamics of generalized tachyon field with different interaction term 


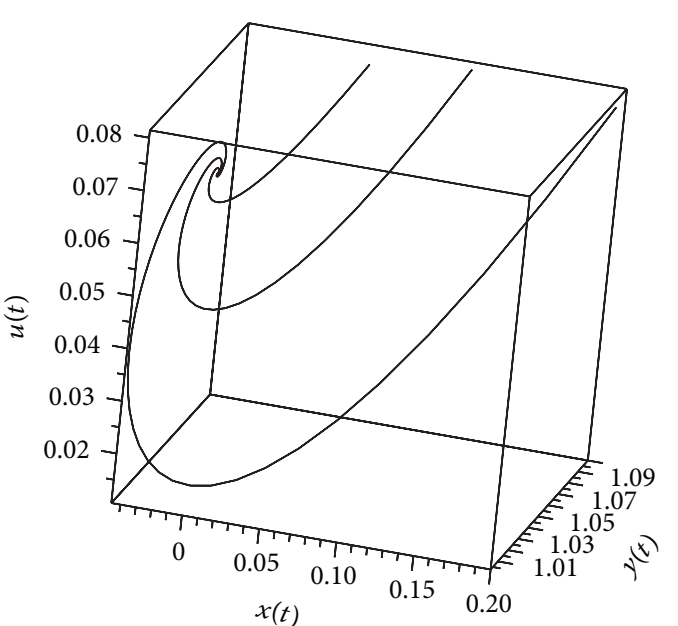

(a)

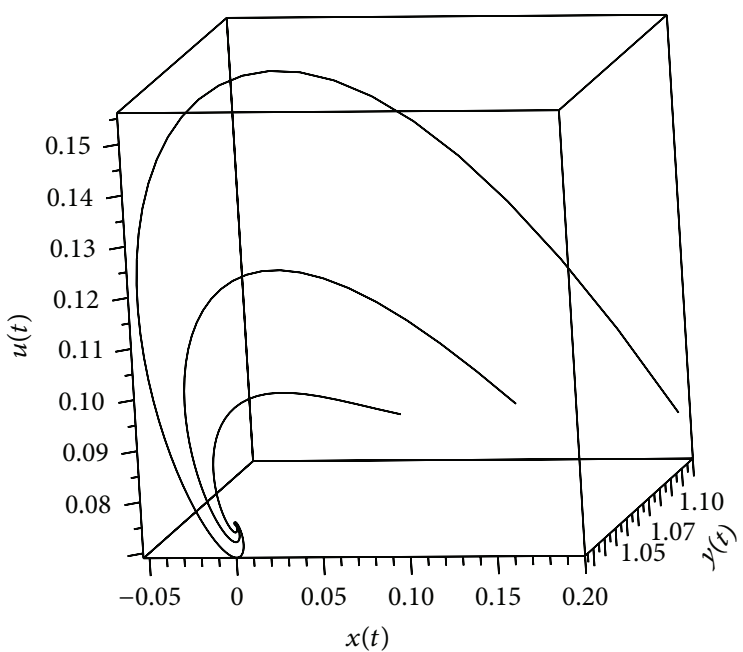

(b)

Figure 3: Three-dimensional phase-space trajectories of the model for $Q=0$ with stable attractors $A_{1}$ (a) and $A_{2}$ (b). The values of the parameters are those mentioned in Figures 1 and 2, respectively.

Q. Although one can work with a general $\gamma(\gamma=1$ and $4 / 3$ correspond to dust matter and radiation, resp.) without loss of generality, we assume $\gamma=1$ for simplicity.

3.1. The Case for $Q=0$. The first case $Q=0$ clearly means that there is no interaction between dark energy and background matter. In this case, there are two critical points presented in Table 1. From (12) and (14) one can obtain the corresponding values of density parameter $\Omega_{\phi}$ and equation of state of dark energy $\omega_{\phi}$ at each point. Also, using (17) we can find the condition required for acceleration $(q<0)$ at each point. These parameters and conditions have been shown in Table 1 . The stability and existence conditions of critical points $A_{1}$ and $A_{2}$ are presented in Table 2. We mention that the corresponding eigenvalues of perturbation matrix $M$ at critical points $A_{1}$ and $A_{2}$ are considerably involved and here we do not present their explicit expressions, but we can find sign of them numerically.

Critical Point $A_{1}$. This critical point is a scaling attractor if $\xi>0, \alpha<0$, and $\lambda<0$. Therefore, cosmological coincidence problem can be alleviated at this point. $A_{1}$ is a saddle point for $\alpha>0$ and $\xi / \lambda>0$.

Critical Point $A_{2} . A_{2}$ can also be a scaling attractor of the model or a saddle point under the same conditions as for $A_{1}$.

In Figure 1 we have chosen the values of the parameters $\xi, \lambda$, and $\alpha$, such that $A_{1}$ became a stable attractor of the model. Plots in Figure 1 show the phase-space trajectories on $x-y, x-u$, and $u-y$ planes from (a) to (c), respectively. The same plots are shown in Figure 2 for critical point $A_{2}$. Note that the values of the parameter have been chosen in the way that $A_{2}$ became a stable point of the model. In Figure 3, the corresponding 3-dimensional phase-space trajectories of the model have been presented. One can see that $A_{1}$ and $A_{2}$ are stable attractors of the model in (a) and (b) plots, respectively.

3.2. The Case for $Q=\beta \kappa \rho_{m} \dot{\phi}$. This deals with the most familiar interaction term extensively considered in the literature (see, e.g., [31, 47-51]). Here $\widehat{Q}$ in terms of auxiliary variables is $\widehat{Q}=\sqrt{3} \beta y^{-1} \Omega_{m}$. Inserting such an interaction term in (18) and setting the left hand sides of the equations to zero lead to the critical points $B_{1}, B_{2}, B_{3}$, and $B_{4}$ presented in Table 3 . In the same table we have provided the corresponding values of $\Omega_{\phi}$ and $\omega_{\phi}$ as well as the condition needed for accelerating universe at each fixed points.

The stability and existence conditions for each point are presented in Table 4. Since the corresponding eigenvalues of the fixed points are complicated we do not give them here, but one can obtain their signs numerically and then examine the stability properties of the critical points.

Critical Point $B_{1}$. This point exists for $\lambda>0$ and $\xi \geq \lambda^{2} / 8 \alpha^{2}$. However, it is an unstable saddle point.

Critical Point $B_{2}$. The critical point $B_{2}$ exists for $\lambda>0$ and $\xi<$ 0 or $\xi \geq \lambda^{2} / 8 \alpha^{2}$. This point is a scaling attractor of the model if $\alpha>0$ and $\xi>0$. Figure 4 shows clearly such a behavior of the model for suitable choices of $\xi, \lambda$, and $\alpha$.

Critical Point $B_{3}$. This point exists for negative values of $\lambda$ and $\xi$ or when $\xi \geq \lambda^{2} / 8 \alpha^{2}$. Also, it is a stable point if $\alpha<0$ and $\xi>0$ and a saddle point if $\alpha>0$ and $\xi<0$. The values of parameters have been chosen in Figure 5 such that 


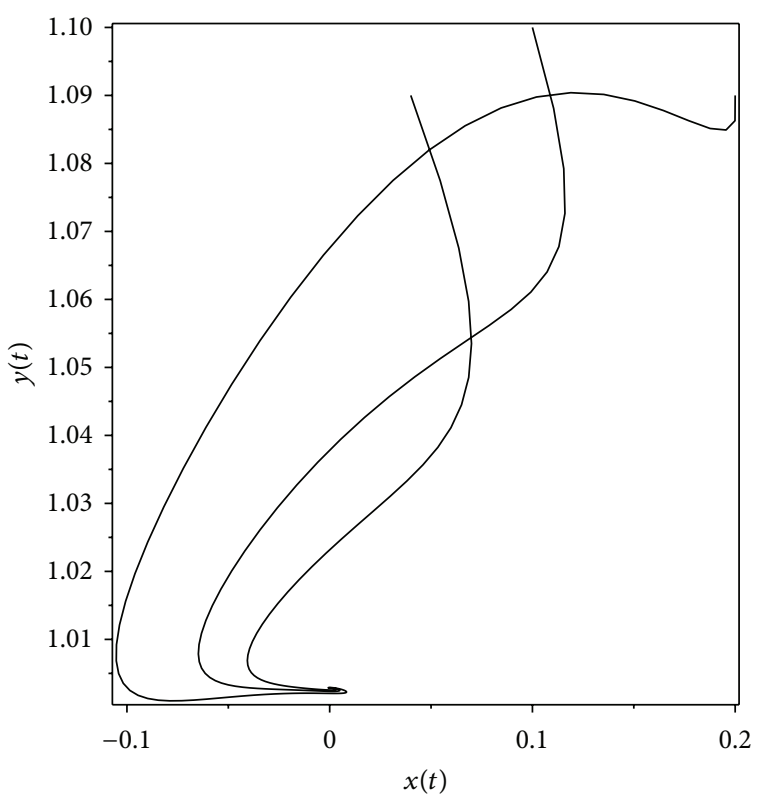

(a)

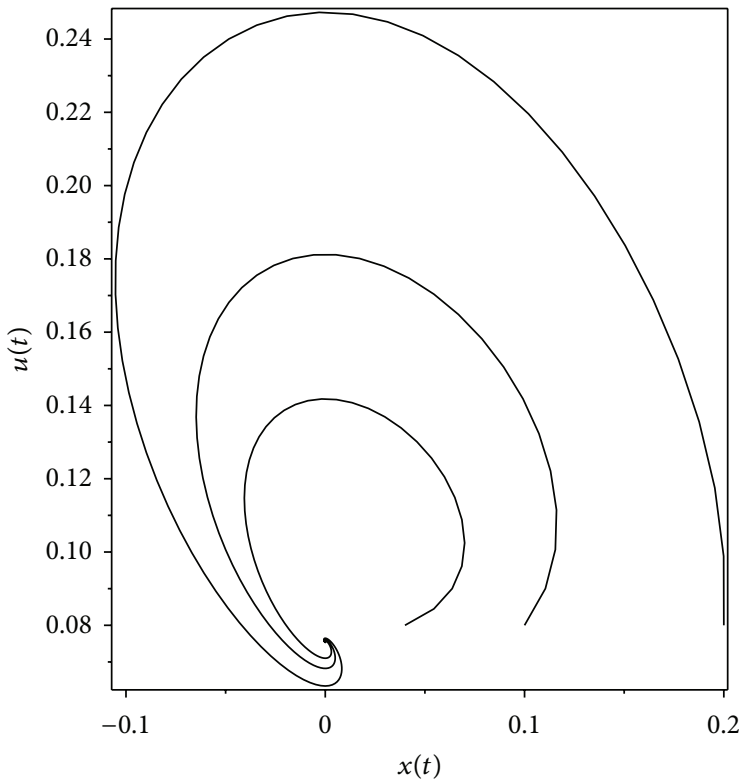

(b)

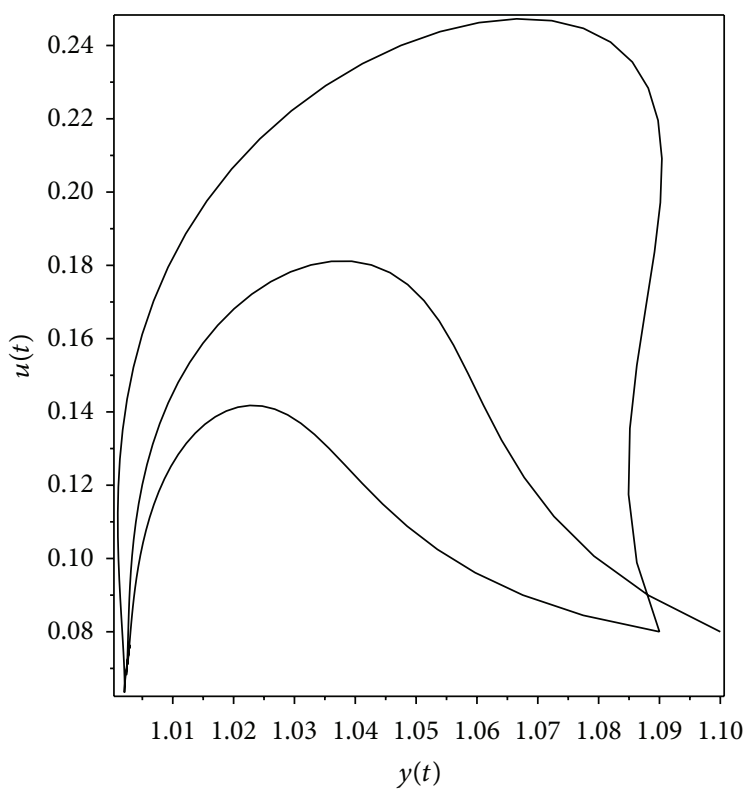

(c)

Figure 4: From (a) to (c), the projections of the phase-space trajectories on the $x-y, x-u$, and $u-y$ planes with $\xi=0.5, \lambda=0.6, \alpha=2$, and $\beta=1.5$ for $Q=\beta \kappa \rho_{m} \dot{\phi}$. For these values of the parameters, point $B_{2}$ is a stable attractor of the model.

$B_{3}$ became a attractor of the model as it is clear from phasespace trajectories.

Critical Point $B_{4}$. The point $B_{4}$ exists for $\lambda<0$ and $\xi \geq \lambda^{2} / 8 \alpha^{2}$. It is a stable point if $\alpha<0$ and $\xi>0$. In Figure 6 values of the parameters $\xi$ and $\alpha$ are those satisfying these constraints and so $B_{4}$ becomes a attractor point for phase-plane trajectories. The corresponding 3-dimensional phase-space trajectories of the model for attractor points $B_{2}$ (a), $B_{3}$ (b), and $B_{4}$ (c) are plotted in Figure 7.

\section{Conclusion}

A model of dark energy with nonminimal coupling of quintessence scalar field with gravity in the framework of teleparallel gravity was called teleparallel dark energy [27]. If one replaces quintessence by tachyon field in such a model, then tachyonic teleparallel dark energy will be constructed [32].

Moreover, although dark energy and dark matter scale differently with the expansion of our universe, according to 


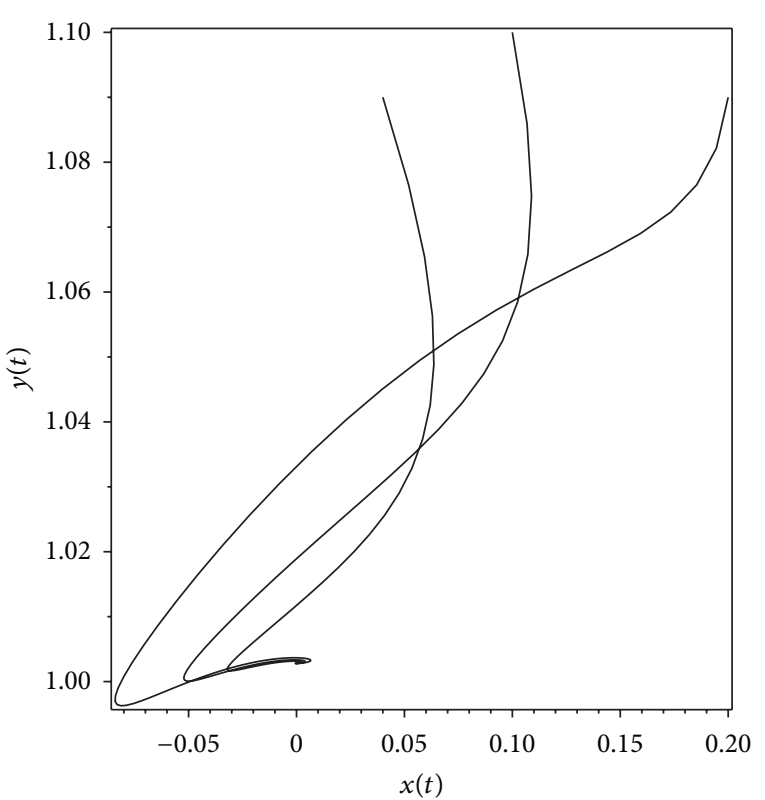

(a)

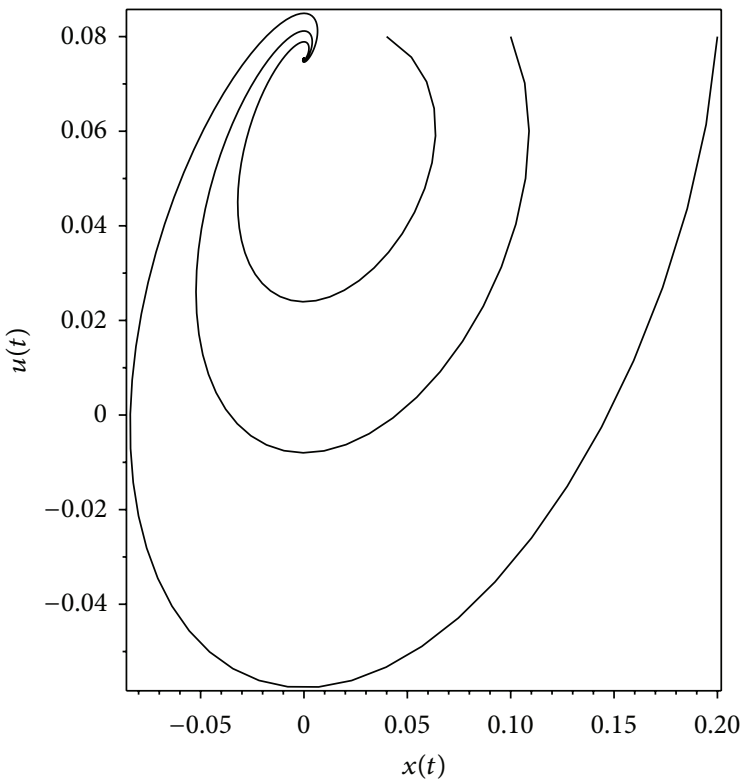

(b)

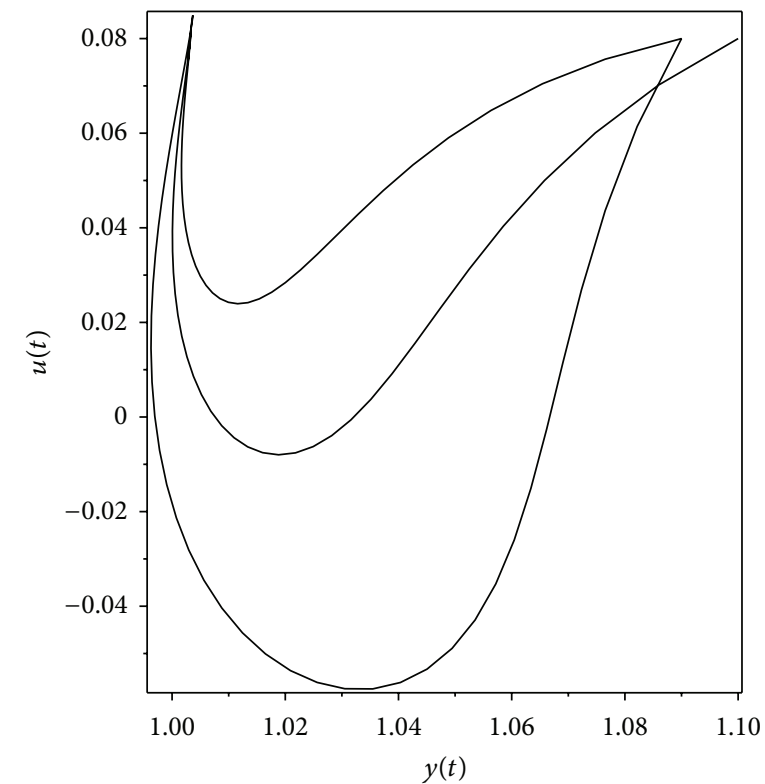

(c)

FiguRE 5: From (a) to (c), the projections of the phase space trajectories on the $x-y, x-u$, and $u-y$ planes with $\xi=0.5, \lambda=-0.6, \alpha=-2$, and $\beta=1.5$ for $Q=\beta \kappa \rho_{m} \dot{\phi}$. For these values of the parameters, point $B_{3}$ is a stable attractor of the model.

the observations [52-54] we are living in an epoch in which dark energy and dark matter densities are comparable and this is the well-known cosmological coincidence problem [30]. This problem can be alleviated in most dark energy models via the method of scaling solutions in which the density parameters of dark energy and dark matter are both nonvanishing over there.

In this paper, we investigated the phase-space analysis of generalized tachyon cosmology in the framework of teleparallel gravity. Our model was described by action (2) which generalizes tachyonic teleparallel dark energy model proposed in [32]. We found some scaling attractors in our model for the case $n=2$ and the coupling function $f(\phi) \propto \phi^{2}$. These scaling attractors are $A_{1}$ and $A_{2}$ when there is no interaction between dark energy and dark matter. $B_{2}, B_{3}$, and $B_{4}$ are scaling attractors in the case that dark energy interacts with dark matter through the interacting term $Q=\beta \kappa \rho_{m} \dot{\phi}$. As it is shown in [29] original teleparallel dark energy model also has a late-time attractor in which dark energy behaves like a cosmological constant and so 


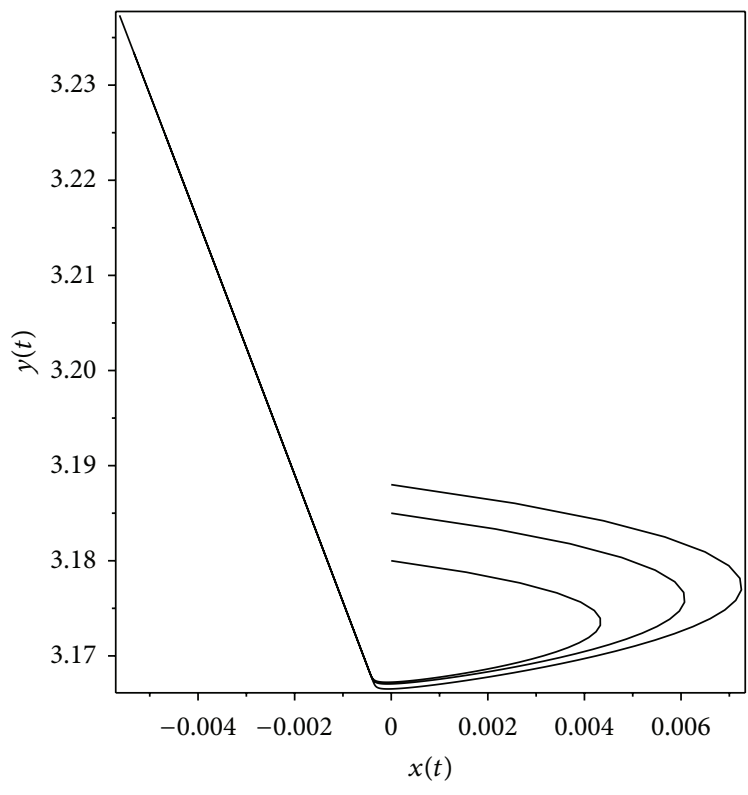

(a)

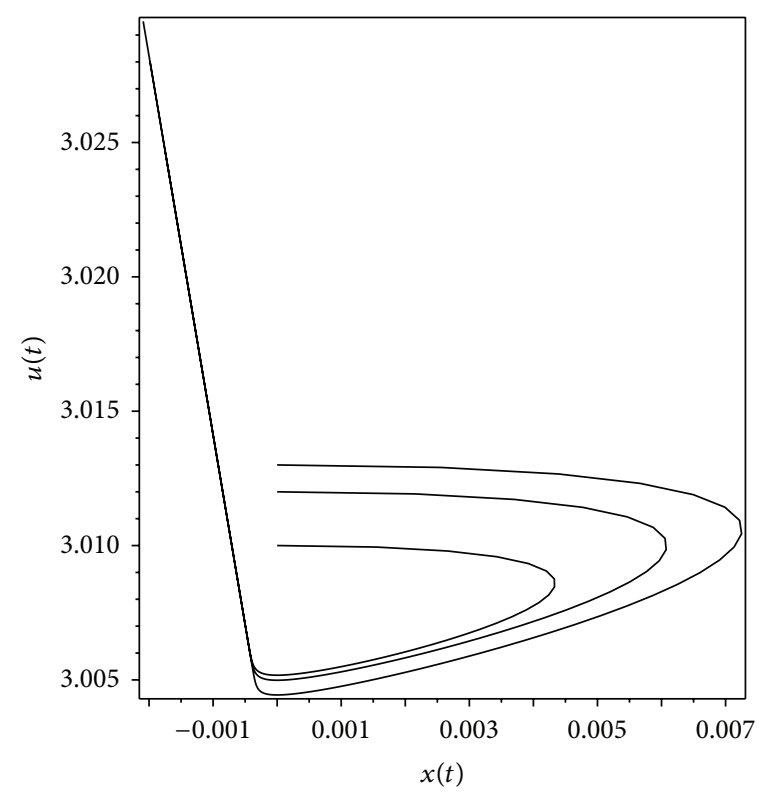

(b)



(c)

FiguRE 6: From (a) to (c), the projections of the phase space trajectories on the $x-y, x-u$, and $u-y$ planes with $\xi=0.5, \lambda=-0.6, \alpha=-0.5$, and $\beta=1.5$ for $Q=\beta \kappa \rho_{m} \dot{\phi}$. For these values of the parameters, point $B_{4}$ is a stable attractor of the model.

it provides a natural way for the stabilization of the dark energy equation of state to the cosmological constant value, without the need for parameter tuning. Also, the authors in [55] by using power-law potentials and nonminimal coupling functions have shown that teleparallel gravity has no stable future solutions for positive nonminimal coupling, and the scalar field results always in oscillations. Our results show that generalized tachyon field represents interesting cosmological behavior in comparison with ordinary tachyon fields in the framework of teleparallel gravity because there is no scaling attractor in the latter model. Note that however in [33] Otalora has considered a dynamically changing coefficient $\alpha=f_{, \phi} / \sqrt{f}$ and found a field matter dominated solution in which it has some portions of the dark energy density in the matter dominated era. So, generalized tachyon field gives us the hope that cosmological coincidence problem can be alleviated without fine-tunings. One can study our model for different kinds of potential and other famous interaction term between dark energy and dark matter. 


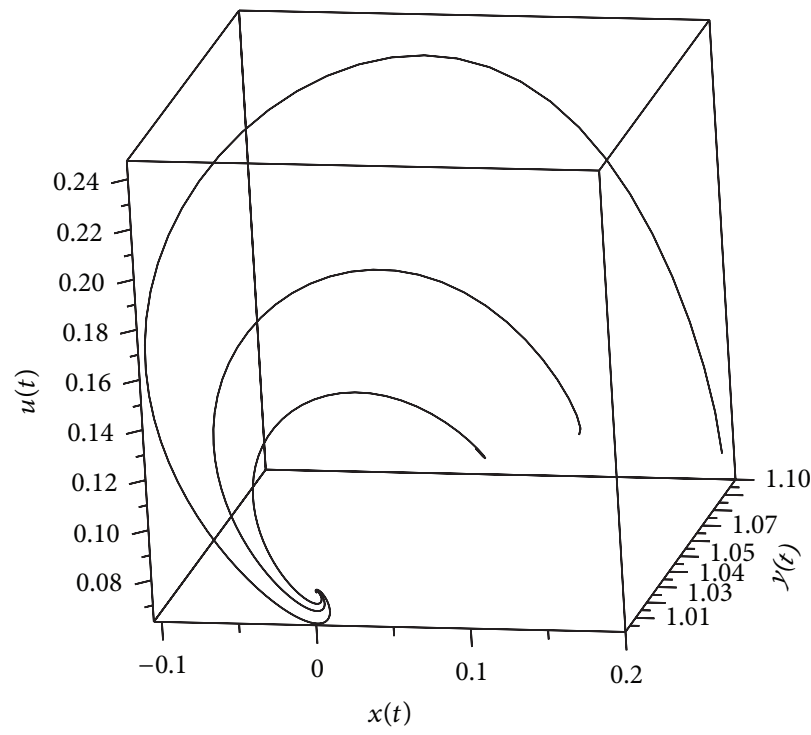

(a)

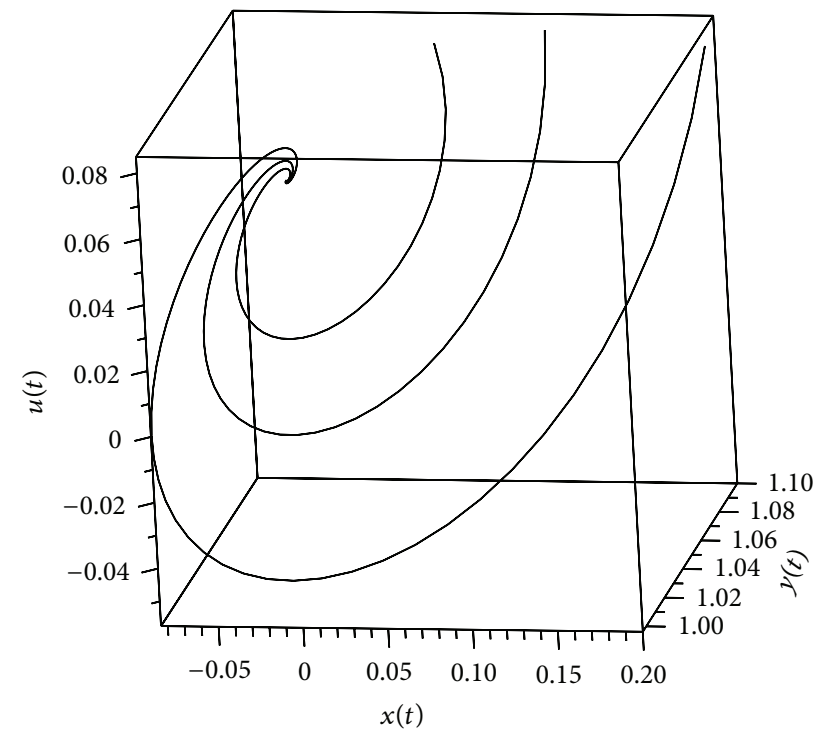

(b)



(c)

Figure 7: Three-dimensional phase-space trajectories of the model for $Q=\beta \kappa \rho_{m} \dot{\phi}$ with stable attractors $B_{2}$ (a), $B_{3}$ (b), and $B_{4}$ (c). The values of the parameters are those mentioned in Figures 4, 5, and 6, respectively.

TABLE 3: Location of the critical points and the corresponding values of the dark energy density parameter $\Omega_{\phi}$ and equation of state $\omega_{\phi}$ and the condition required for an accelerating universe for $Q=\beta \kappa \rho_{m} \dot{\phi}$. Here $\theta_{1}=\left(4 \alpha \xi+\sqrt{16 \alpha^{2} \xi^{2}-2 \lambda^{2} \xi}\right) / 2 \lambda \xi$ and $\theta_{2}=\left(4 \alpha \xi-\sqrt{16 \alpha^{2} \xi^{2}-2 \lambda^{2} \xi}\right) / 2 \lambda \xi$.

\begin{tabular}{lcccc}
\hline Label & $\left(x_{c}, y_{c}, u_{c}\right)$ & $\Omega_{\phi}$ & $\omega_{\phi}$ & Acceleration \\
\hline$B_{1}$ & $0, \frac{2 \sqrt{2 \lambda \alpha \xi \theta_{1}}}{\lambda}, \theta_{1}$ & $2 \xi \theta_{1}\left(\frac{4 \alpha}{\lambda}-\theta_{1}\right)$ & $\frac{2\left(\xi \theta_{1}^{3}+2 \alpha / \lambda\right)}{\left(\theta_{1}-4 \alpha / \lambda\right)\left(1+2 \xi \theta_{1}^{2}\right)}$ & $\lambda>\frac{8 \alpha\left(\xi \theta_{1}^{2}-1\right)}{\theta_{1}\left(8 \xi \theta_{1}^{2}+1\right)}$ \\
\hline$B_{2}$ & $0, \frac{2 \sqrt{2 \lambda \alpha \xi \theta_{2}}}{\lambda}, \theta_{2}$ & $\frac{2\left(\xi \theta_{2}^{3}+2 \alpha / \lambda\right)}{\left(\theta_{2}-4 \alpha / \lambda\right)\left(1+2 \xi \theta_{2}^{2}\right)}$ & $\lambda>\frac{8 \alpha\left(\xi \theta_{2}^{2}-1\right)}{\theta_{2}\left(8 \xi \theta_{2}^{2}+1\right)}$ \\
\hline$B_{3}$ & $0,-\frac{2 \sqrt{2 \lambda \alpha \xi \theta_{1}}}{\lambda}, \theta_{1}\left(\frac{4 \alpha}{\lambda}-\theta_{2}\right)$ & $\frac{2\left(\xi \theta_{1}^{3}+2 \alpha / \lambda\right)}{\left(\theta_{1}-4 \alpha / \lambda\right)\left(1+2 \xi \theta_{1}^{2}\right)}$ & $\lambda>\frac{8 \alpha\left(\xi \theta_{1}^{2}-1\right)}{\theta_{1}\left(8 \xi \theta_{1}^{2}+1\right)}$ \\
\hline$B_{4}$ & $0,-\frac{2 \sqrt{2 \lambda \alpha \xi \theta_{2}}}{\lambda}, \theta_{2}$ & $2 \xi \theta_{1}\left(\frac{4 \alpha}{\lambda}-\theta_{1}\right)$ & $\frac{2\left(\xi \theta_{2}^{3}+2 \alpha / \lambda\right)}{\left(\theta_{2}-4 \alpha / \lambda\right)\left(1+2 \xi \theta_{2}^{2}\right)}$ & $\lambda>\frac{8 \alpha\left(\xi \theta_{2}^{2}-1\right)}{\theta_{2}\left(8 \xi \theta_{2}^{2}+1\right)}$ \\
\hline
\end{tabular}


TABLE 4: Stability and existence conditions of the critical points of the model for $Q=\beta \kappa \rho_{m} \dot{\phi}$.

\begin{tabular}{lcc}
\hline Label & Stability & Existence \\
\hline$B_{1}$ & Saddle point & $\lambda>0$ and $\xi \geq \lambda^{2} / 8 \alpha^{2}$ \\
\hline & if $\alpha>0$ and $\xi>0$ & $\lambda>0$ and \\
$B_{2}$ & Saddle point & for all $\xi<0$ \\
& if $\alpha<0$ and $\xi<0$ and & or \\
stable point & $\xi \geq \lambda^{2} / 8 \alpha^{2}$ \\
\hline & if $\alpha>0$ and $\xi>0$ & $\lambda<0$ and \\
$B_{3}$ & Saddle point & for all $\xi<0$ \\
& if $\alpha>0$ and $\xi<0$ and & or \\
\hline$B_{4}$ & stable point & $\xi \geq \lambda^{2} / 8 \alpha^{2}$ \\
\hline & if $\alpha<0$ and $\xi>0$ & $\lambda<0$ and $\xi \geq \lambda^{2} / 8 \alpha^{2}$ \\
\hline
\end{tabular}

\section{Appendix}

\section{Perturbation Matrix Elements}

The elements of $3 \times 3$ matrix $M$ of the linearized perturbation equations for the real and physically meaningful critical points $\left(x_{c}, y_{c}, u_{c}\right)$ of the autonomous system (18) read as follows:

$$
\begin{aligned}
& M_{11}=3 v_{c}^{2}\left(\frac{\sqrt{3}}{2} \lambda x_{c} y_{c}\left(2 x_{c}^{2}+\nu_{c}^{2}\left(1+3 x_{c}^{4}\right)\right)-6 \mu_{c}^{-2} \nu_{c}^{2} x_{c}^{2}\right. \\
& \left.-4 \sqrt{3} \alpha \xi u_{c} x_{c} \nu_{c}^{2} y_{c}^{-1}\right)+\sqrt{3} \lambda x_{c} y_{c}-3+\mathscr{M}_{11}, \\
& M_{12}=\frac{\sqrt{3}}{4}\left(\lambda\left(2 x_{c}^{2}+v_{c}^{2}\left(1+3 x_{c}^{4}\right)\right)+8 \alpha \xi u_{c} v_{c}^{2} y_{c}^{-2}\right)+\mathscr{M}_{12} \\
& M_{13}=-2 \sqrt{3} \alpha \xi v_{c}^{2} y_{c}^{-1}+\mathscr{M}_{13} \\
& M_{21}=\frac{2 y_{c}^{2}\left(\sqrt{3} \alpha \xi u_{c}+3 x_{c} y_{c} \nu_{c}^{-2}\right)}{\left(2 \xi u_{c}^{2}+1\right)}-\frac{\sqrt{3} \lambda y_{c}^{2}}{2}, \\
& M_{22}=\frac{2 y_{c}\left(-(9 / 4) \mu_{c}^{-4} y_{c}+5 \sqrt{3} \alpha \xi x_{c} u_{c}\right)}{\left(2 \xi u_{c}^{2}+1\right)}-\sqrt{3} \lambda x_{c} y_{c}+\frac{3}{2} \text {, } \\
& M_{23}=\frac{6 \xi u_{c} y_{c}^{2}\left(-(10 \sqrt{3} / 3) \alpha \xi u_{c} x_{c}+\mu_{c}^{-4} y_{c}^{2}\right)}{\left(2 \xi u_{c}^{2}+1\right)^{2}} \\
& +\frac{5 \sqrt{3} \alpha \xi x_{c} y_{c}^{2}}{2 \xi u_{c}^{2}+1} \\
& M_{31}=\frac{\sqrt{3} \alpha y_{c}}{2} \\
& M_{32}=\frac{\sqrt{3} \alpha x_{c}}{2} \\
& M_{33}=0 \text {, }
\end{aligned}
$$

where in the case of $Q=0$ we have $\mathscr{M}_{11}=\mathscr{M}_{12}=\mathscr{M}_{13}=0$ and in the case of $Q=\beta \kappa \rho_{m} \dot{\phi}$, we have

$$
\begin{aligned}
& \mathscr{M}_{11}=4 \sqrt{3} \beta \nu_{c}^{-2} x_{c} y_{c}, \\
& \mathscr{M}_{12}=2 \sqrt{3} \beta \mu_{c}^{-2}\left(1+3 x_{c}^{2}\right), \\
& \mathscr{M}_{13}=-4 \sqrt{3} \beta \xi u_{c} y_{c}^{-1} .
\end{aligned}
$$

Examining the eigenvalues of the matrix $M$ for each critical point, one determines its stability conditions.

\section{Conflict of Interests}

The authors declare that there is no conflict of interests regarding the publication of this paper.

\section{References}

[1] S. Weinberg, "The cosmological constant problem," Reviews of Modern Physics, vol. 61, no. 1, pp. 1-23, 1989.

[2] S. M. Carroll, "The cosmological constant," Living Reviews in Relativity, vol. 4, article 1, 2001.

[3] R.-J. Yang and S. N. Zhang, "The age problem in the $\Lambda$ CDM model," Monthly Notices of the Royal Astronomical Society, vol. 407, no. 3, pp. 1835-1841, 2010.

[4] Y.-F. Cai, E. N. Saridakis, M. R. Setare, and J.-Q. Xia, “Quintom cosmology: theoretical implications and observations," Physics Reports, vol. 493, no. 1, pp. 1-60, 2010.

[5] K. Bamba, S. Capozziello, S. Nojiri, and S. D. Odintsov, "Dark energy cosmology: the equivalent description via different theoretical models and cosmography tests," Astrophysics and Space Science, vol. 342, no. 1, pp. 155-228, 2012.

[6] A. Sen, "Remarks on tachyon driven cosmology," Physica Scripta, vol. 2005, no. 117, article 70, 2005.

[7] G. W. Gibbons, "Cosmological evolution of the rolling tachyon," Physics Letters B, vol. 537, no. 1-2, pp. 1-4, 2002.

[8] M. R. Garousi, "Tachyon couplings on non-BPS D-branes and Dirac-Born-Infeld action," Nuclear Physics B, vol. 584, no. 1-2, pp. 284-299, 2000.

[9] A. Sen, "Field theory of tachyon matter," Modern Physics Letters A, vol. 17, no. 27, pp. 1797-1804, 2002.

[10] A. Unzicker and T. Case, “Translation of Einstein's attempt of a unified field theory with teleparallelism," http://arxiv.org/abs/ physics/0503046.

[11] K. Hayashi and T. Shirafuji, "New general relativity," Physical Review D, vol. 19, pp. 3524-3553, 1979, Addendum in Physical Review D, vol. 24, pp. 3312-3314, 1982.

[12] R. Aldrovandi and J. G. Pereira, Teleparallel Gravity: An Introduction, Springer, Dordrecht, Netherlands, 2013.

[13] J. W. Maluf, “The teleparallel equivalent of general relativity," Annalen der Physik, vol. 525, no. 5, pp. 339-357, 2013.

[14] R. Ferraro and F. Fiorini, "Modified teleparallel gravity: inflation without an inflaton," Physical Review D: Particles, Fields, Gravitation, and Cosmology, vol. 75, no. 8, Article ID 084031, 2007.

[15] G. R. Bengochea and R. Ferraro, "Dark torsion as the cosmic speed-up," Physical Review D: Particles, Fields, Gravitation, and Cosmology, vol. 79, Article ID 124019, 2009.

[16] E. V. Linder, "Einstein's other gravity and the acceleration of the Universe," Physical Review D, vol. 81, Article ID 127301, 2010. 
[17] A. de Felice and S. Tsujikawa, "f (R) theories," Living Reviews in Relativity, vol. 13, no. 3, pp. 1-161, 2010.

[18] S. Nojiri and S. D. Odintsov, "Unified cosmic history in modified gravity: from $\mathrm{F}(\mathrm{R})$ theory to Lorentz non-invariant models," Physics Reports, vol. 505, no. 2-4, pp. 59-144, 2011.

[19] B. L. Spokoiny, "Inflation and generation of perturbations in broken-symmetric theory of gravity," Physics Letters B, vol. 147, no. 1-3, pp. 39-43, 1984.

[20] F. Perrotta, C. Baccigalupi, and S. Matarrese, "Extended quintessence," Physical Review D, vol. 61, Article ID 023507, 1999.

[21] V. Faraoni, "Inflation and quintessence with nonminimal coupling," Physical Review D, vol. 62, no. 2, Article ID 023504, 15 pages, 2000.

[22] E. Elizalde, S. Nojiri, and S. D. Odintsov, "Late-time cosmology in a (phantom) scalar-tensor theory: dark energy and the cosmic speed-up," Physical Review D, vol. 70, Article ID 043539, 2004.

[23] O. Hrycyna and M. Szydlowski, "Non-minimally coupled scalar field cosmology on the phase plane," Journal of Cosmology and Astroparticle Physics, vol. 2009, article 026, 2009.

[24] O. Hrycyna and M. Szydlowski, "Extended Quintessence with non-minimally coupled phantom scalar field," Physical Review D: Particles, Fields, Gravitation, and Cosmology, vol. 76, Article ID 123510, 2007.

[25] R. C. de Souza and G. M. Kremer, "Constraining non-minimally coupled tachyon fields by the Noether symmetry," Classical and Quantum Gravity, vol. 26, no. 13, Article ID 135008, 2009.

[26] A. A. Sen and N. C. Devi, "Cosmology with non-minimally coupled k-field," General Relativity and Gravitation, vol. 42, no. 4, pp. 821-838, 2010.

[27] C.-Q. Geng, C.-C. Lee, E. N. Saridakis, and Y.-P. Wu, “Teleparallel' dark energy," Physics Letters, Section B: Nuclear, Elementary Particle and High-Energy Physics, vol. 704, no. 5, pp. 384-387, 2011.

[28] C.-Q. Geng, C.-C. Lee, and E. N. Saridakis, "Observational constraints on teleparallel dark energy," Journal of Cosmology and Astroparticle Physics, vol. 2012, no. 1, article 002, 2012.

[29] C. Xu, E. N. Saridakis, and G. Leon, "Phase-space analysis of teleparallel dark energy," Journal of Cosmology and Astroparticle Physics, vol. 2012, no. 7, article 5, 2012.

[30] H. Wei, "Dynamics of teleparallel dark energy," Physics Letters $B$, vol. 712, no. 4-5, pp. 430-436, 2012.

[31] G. Otalora, "Scaling attractors in interacting teleparallel dark energy," Journal of Cosmology and Astroparticle Physics, vol. 2013, no. 7, article 44, 2013.

[32] A. Banijamali and B. Fazlpour, "Tachyonic teleparallel dark energy," Astrophysics and Space Science, vol. 342, no. 1, pp. 229235, 2012.

[33] G. Otalora, "Cosmological dynamics of tachyonic teleparallel dark energy," Physical Review D, vol. 88, no. 6, Article ID 063505, 8 pages, 2013.

[34] S. H. Pereira, S. S. A. Pinho, and J. M. H. da Silva, "Some remarks on the attractor behaviour in ELKO cosmology," Journal of Cosmology and Astroparticle Physics, vol. 2014, article 020, 2014.

[35] O. Bertolami, F. Gil Pedro, and M. Le Delliou, "Dark energydark matter interaction and putative violation of the equivalence principle from the Abell cluster A586," Physics Letters B, vol. 654, no. 5-6, pp. 165-169, 2007.

[36] O. Bertolami, F. Gil Pedro, and M. le Delliou, "The Abell cluster A586 and the detection of violation of the equivalence principle," General Relativity and Gravitation, vol. 41, no. 12, pp. 2839-2846, 2009.

[37] M. Le Delliou, O. Bertolami, and F. Gil Pedro, "Dark energydark matter interaction from the abell cluster A586 and violation of the equivalence principle," AIP Conference Proceedings, vol. 957, p. 421, 2007.

[38] O. Bertolami, F. G. Pedro, and M. L. Delliou, "Dark energydark matter interaction from the abell cluster A586," European Astronomical Society Publications Series, vol. 30, pp. 161-167, 2008.

[39] E. Abdalla, L. R. Abramo, L. Sodre, and B. Wang, "Signature of the interaction between dark energy and dark matter in galaxy clusters," Physics Letters B, vol. 673, pp. 107-110, 2009.

[40] E. Abdalla, L. R. Abramo, and J. C. C. de Souza, "Signature of the interaction between dark energy and dark matter in observations," Physical Review D, vol. 82, Article ID 023508, 2010.

[41] N. Banerjee and S. Das, "Acceleration of the universe with a simple trigonometric potential," General Relativity and Gravitation, vol. 37, no. 10, pp. 1695-1703, 2005.

[42] S. Das and N. Banerjee, "An interacting scalar field and the recent cosmic acceleration," General Relativity and Gravitation, vol. 38, no. 5, pp. 785-794, 2006.

[43] S. Das and N. Banerjee, "Brans-Dicke scalar field as a chameleon," Physical Review D, vol. 78, Article ID 043512, 2008.

[44] S. Unnikrishnan, "Can cosmological observations uniquely determine the nature of dark energy?" Physical Review D, vol. 78, Article ID 063007, 2008.

[45] R. Yang and J. Qi, "Dynamics of generalized tachyon field," The European Physical Journal C, vol. 72, article 2095, 2012.

[46] I. Quiros, T. Gonzalez, D. Gonzalez, Y. Napoles, R. GarcíaSalcedo, and C. Moreno, "A study of tachyon dynamics for broad classes of potentials," Classical and Quantum Gravity, vol. 27, no. 21, Article ID 215021, 2010.

[47] E. J. Copeland, A. R. Liddle, and D. Wands, "Exponential potentials and cosmological scaling solutions," Physical Review $D$, vol. 57, no. 8, pp. 4686-4690, 1998.

[48] L. Amendola, "Scaling solutions in general nonminimal coupling theories," Physical Review D, vol. 60, Article ID 043501, 1999.

[49] Z. K. Guo, R. G. Cai, and Y. Z. Zhang, "Cosmological evolution of interacting phantom energy with dark matter," Journal of Cosmology and Astroparticle Physics, vol. 2005, article 002, 2005.

[50] H. Wei, "Cosmological evolution of quintessence and phantom with a new type of interaction in dark sector," Nuclear Physics B, vol. 845, no. 3, pp. 381-392, 2011.

[51] L. P. Chimento, A. S. Jakubi, D. Pavón, and W. Zimdahl, "Interacting quintessence solution to the coincidence problem," Physical Review D, vol. 67, no. 8, Article ID 083513, 2003.

[52] E. J. Copeland, M. Sami, and S. Tsujikawa, "Dynamics of dark energy," International Journal of Modern Physics, D: Gravitation, Astrophysics, Cosmology, vol. 15, no. 11, pp. 1753-1935, 2006.

[53] J. A. Frieman, M. S. Turner, and D. Huterer, "Dark energy and the accelerating universe," Annual Review of Astronomy and Astrophysics, vol. 46, pp. 385-432, 2008.

[54] S. Tsujikawa, "Dark energy: investigation and modeling," in A Challenge for Modern Cosmology, vol. 370 of Astrophysics and Space Science Library, pp. 331-402, Springer, Dordrecht, Netherlands, 2011.

[55] M. Skugoreva, E. Saridakis, and A. Toporensky, "Dynamical features of scalar-torsion theories," to appear in Physical Review $D$. 

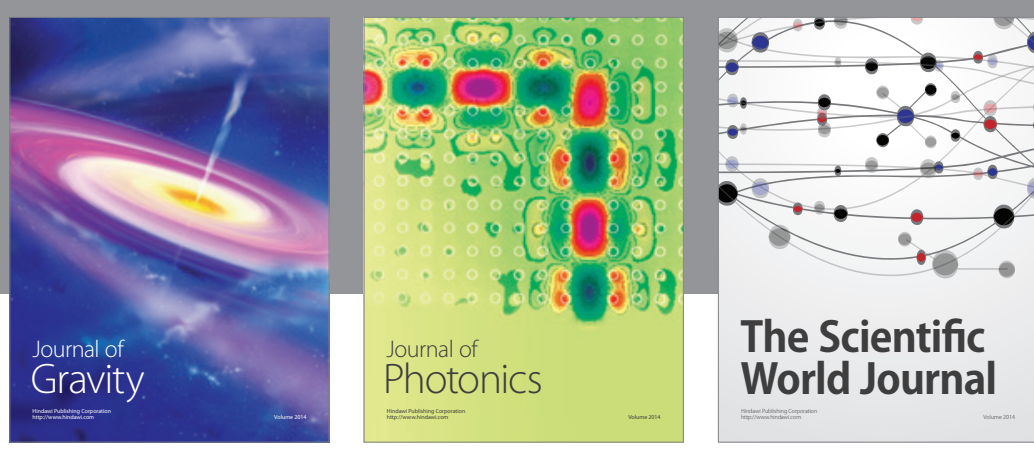

The Scientific World Journal
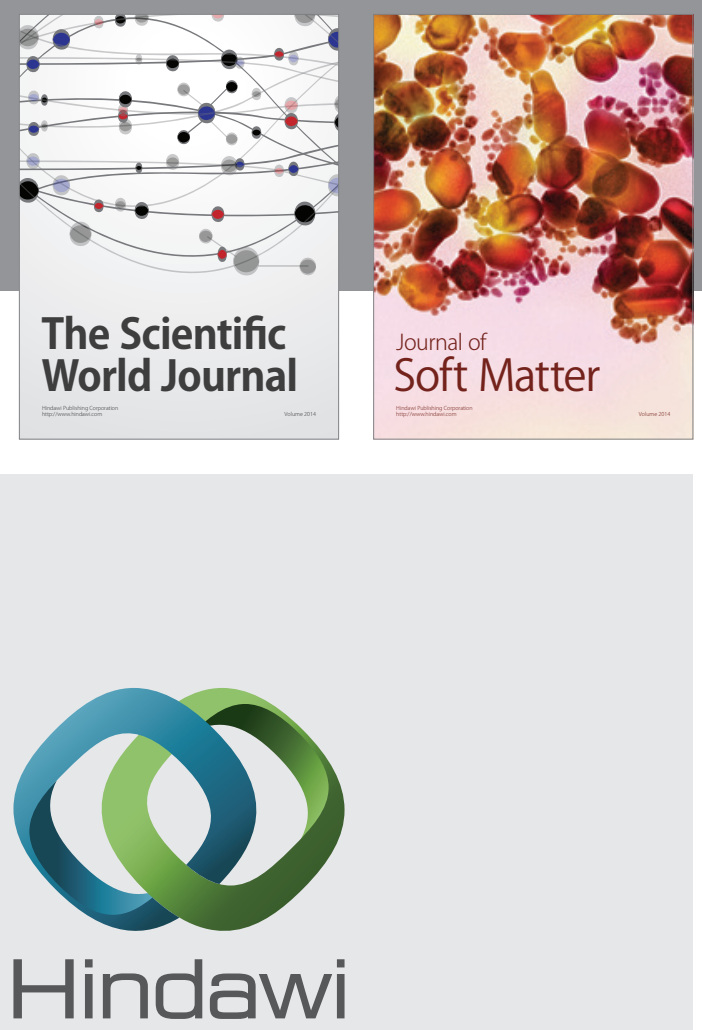

Submit your manuscripts at

http://www.hindawi.com

nternational Journal of

Statistical Mechanics
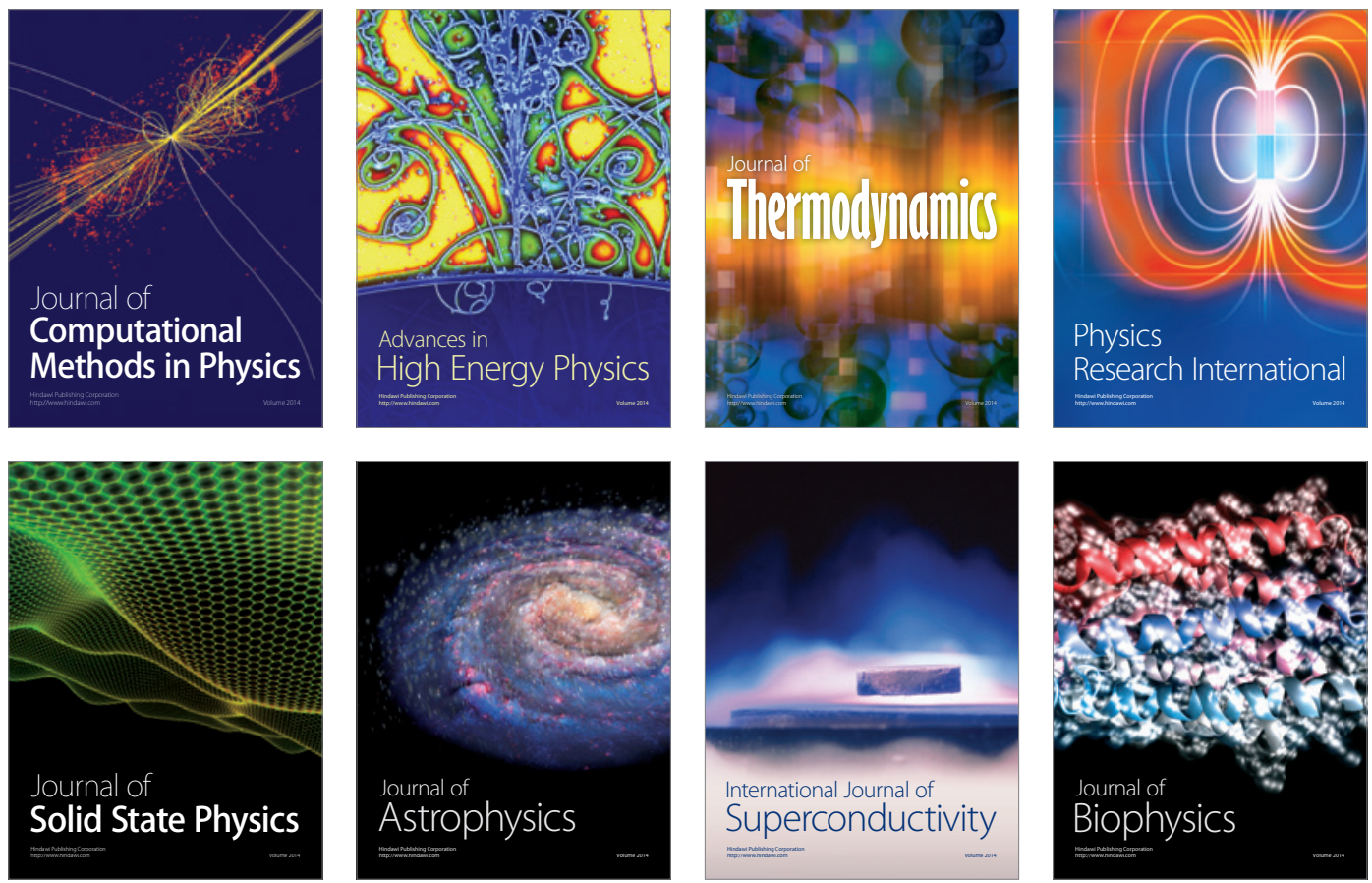
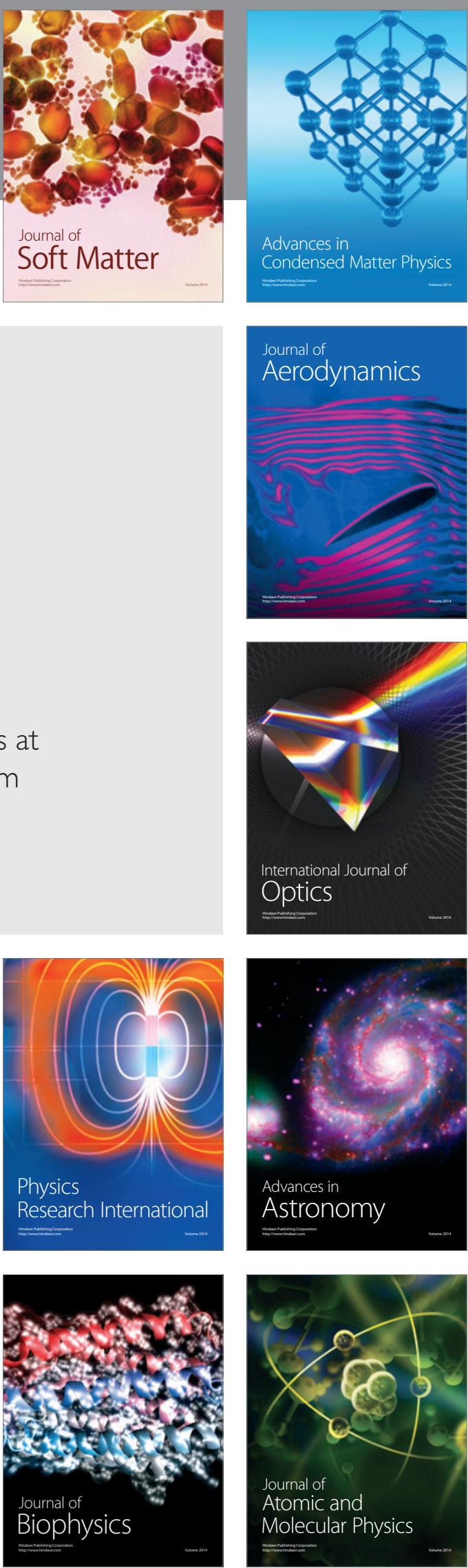